\title{
Diallel analysis of yield components of snap beans exposed to two temperature stress environments
}

\author{
Katy M. Rainey \& Phillip D. Griffiths* \\ Department of Horticultural Sciences, Cornell University NYSAES, Geneva, NY 14456, U.S.A.; \\ (*author for correspondence: e-mail:pdg8@cornell.edu)
}

Received 24 February 2004; accepted 9 January 2005

Key words: abiotic stress, chilling tolerance, combining ability, heat tolerance, Phaseolus vulgaris (L.), yield components

\section{Summary}

Ten snap beans ('Barrier', 'Brio', 'Carson', 'Cornell 502', 'CT 70', 'HB 1880', 'Hystyle', 'Labrador', 'Opus' and 'Venture') were selected for differential temperature tolerance and used as parents in a complete diallel mating design. The $45 F_{1}$ hybrid lines (with reciprocals) and parents were screened at $32{ }^{\circ} \mathrm{C}$ day $/ 28^{\circ} \mathrm{C}$ night, and in a separate experiment, $16^{\circ} \mathrm{C}$ day $/ 10^{\circ} \mathrm{C}$ night, during reproductive development in replicated controlled environments. Variation for yield under temperature treatments was observed among parents and hybrids, with certain hybrids exceeding parental performance. Significant $(P \leq 0.0001)$ general combining ability (GCA), and significant $(P \leq$ 0.05 ) specific combining ability (SCA) were observed for yield components including pod number, seed number, and seeds per pod. There was evidence that pod number and seeds per pod under temperature stress are under separate genetic control. Reciprocal effects and heterosis were not significant. GCA could not be predicted from parental performance. The breeding line 'Cornell 502' had the highest GCA under high temperature, and the cultivar 'Brio' had the highest GCA under low temperature. The cross 'Brio' $\times$ 'Venture' was high yielding in both temperature treatments. Heat tolerance and chilling tolerance were associated in certain parents and hybrids. However, performance under high and low temperature treatments was not generally correlated in the parents and hybrids, indicating that these traits should be selected separately.

\section{Introduction}

Common bean (Phaseolus vulgaris L.) is a warm season annual crop that grows best at moderate temperatures (Hall, 2001). The ideal growing temperature is considered to be $23^{\circ} \mathrm{C}$ (Dickson \& Boettger, 1984a), or between 15 and $27^{\circ} \mathrm{C}$ (Bliss, 1980), with optimal temperatures for pod set between 15 and $25^{\circ} \mathrm{C}$ (Inoue \& Suzuki, 1959). A temperature of $10^{\circ} \mathrm{C}$ is close to the lower limit for growth in common bean (Austin \& Maclean, 1972). Research in common bean on the effects of sub-optimal temperatures has focused on germination, emergence, and seedling growth (Dickson, 1971; Otubo et al., 1996). Very little information is available on the effects of low temperatures during reproductive development. There is evidence that chilling tolerance at juvenile stages of development (germination, emergence, and seedling growth) is under independent genetic control from chilling tolerance during reproductive development (Dickson \& Petzoldt, 1987; Kemp, 1973; Melo et al., 1997). Dickson \& Boettger (1984a) reported night temperatures of 8 and $12^{\circ} \mathrm{C}$ significantly lowered pollen germination in vivo compared to $18^{\circ} \mathrm{C}$. Low night temperatures also reduced seeds per pod, with a combination of low day and night causing the lowest seed set. This reduction was attributed to ovule dysfunction, as pollen appeared normal. Farlow (1981) observed similar results of reduced fertility of the female gamete at $13.9{ }^{\circ} \mathrm{C}$, with undeveloped ovules (i.e. missing seeds) distributed throughout 
pods, and no seed set. Kemp (1973) observed damage or destruction of flower primordial at constant $7{ }^{\circ} \mathrm{C}$. In a separate study, Dickson \& Boettger (1984b), established that controlled environment temperature conditions of $16^{\circ} \mathrm{C}$ day $/ 8{ }^{\circ} \mathrm{C}$ night identified snap beans that were early maturing under low temperatures, and that were also tolerant to low temperature field conditions.

Snap bean is crop for which product quality is important and optimal timing for a single destructive harvest represents a moving target (Myers \& Baggett, 1999). High temperature stress $\left(>30^{\circ} \mathrm{C}\right.$ day and/or $>20^{\circ} \mathrm{C}$ night) during reproductive development reduces both yield and quality in snap bean (Singh, 2001) and is a major production constraint in temperate growing areas. Brief periods of high temperatures cause split sets in snap bean fields due to abscission of reproductive organs, which can be the primary determinant of pod number under heat stress (Ofir et al., 1993). Reciprocal pollinations between heat-stressed plants to plants grown at optimal temperatures indicated that pollen is more affected by heat stress than female reproductive structures (Gross \& Kigel, 1994; Monterroso \& Wien, 1990). High temperatures negatively affect other aspects of reproductive development, such as anther dehiscence, pollen germination, and pollen tube growth. Failure of anther dehiscence occurred with heat treatment during sporogenesis (13-8 days before anthesis [DBA]) in heat tolerant and heat sensitive cultivars (Gross \& Kigel, 1994), or only in heat sensitive lines (Porch \& Jahn, 2001). Gross \& Kigel (1994) observed that the number of pollen tubes penetrating the stigma was reduced with high temperatures applied 7-0 DBA, but that there were still more pollen tubes penetrating the stigma than there were ovules to fertilize. Also, under heat stress conditions, the most likely ovules to develop were those positioned nearest to the blossom end of the pod, this can be attributed to high temperature constraints on pollen tube growth, sensitivity of the gynoecium to high temperatures at anthesis, or both. This result suggested some combination of impaired pollen development, reduction of pollen tube length, failure of fertilization, and embryo abortion is occurring with heat stress in common bean. Also, high temperatures around anthesis disrupt fertilization and ovule development (Gross \& Kigel, 1994; Porch \& Jahn, 2001), causing deformed pods in pods in snap bean due to reduced seed set.

Coincidence of tolerances to high and low temperature stress has been reported in snap beans (Dickson, 1993; Dickson \& Boettger, 1984a), oat [Avena sativa
L.] (Coffman, 1957; Mashiringi \& Harahwa, 1985), and maize [Zea mays L.] (Yacoob \& Filion, 1986). Porch (2001) found that snap bean lines developed for cold tolerance had significant positive general combining ability (GCA) under high temperatures for traits related to heat tolerance. A cowpea line selected for low temperature conditions in Minnesota was also very tolerant to high temperature; however, the correlation may have been related to earliness (Hall, 2004). It is possible that heat tolerant snap bean cultivars and lines may also possess cold tolerance. Correlation of these traits in snap bean material could enhance selection strategies, and provide evidence for yield stability in the materials studied. Evidence for correlation might also provide clues to stress tolerance mechanisms and enhance molecular studies. For example, recent studies have uncovered cross-talk between signaling pathways for plant perception of salt, drought, and cold stresses (Chinnusamy et al., 2004).

Tolerance to high and low temperature stresses is not always associated in bean. Nakano et al. (1997) developed a heat tolerant, black-seeded, indeterminate snap bean breeding line ('Haibushi') from South Asian germplasm, and found it performed poorly under low temperature (monthly mean air temperature of $17^{\circ} \mathrm{C}$ ) compared to standard cultivars. Tolerance to chilling germination and heat tolerance were not correlated in cowpea (El-kholy et al., 1997).

Using a diallel mating design, this study focuses on identifying snap bean cultivars and lines for developing heat tolerant snap bean cultivars, and also examines possible associations between tolerance to high and low temperatures at reproductive development. A previous diallel analyses by Shonnard \& Gepts (1994) showed that additive genetic effects are important contributors to yield under heat stress in dry bean, indicating that improvement of reproductive heat tolerance could be possible through combinations of heat tolerant lines. The snap bean parents used in this study were all large sieve size (4-5), determinate type I growth habit, selfpollinated lines. This approach reduced genetic and phenotypic variability in the parents and hybrids associated with growth habit, maturity, and pod and seed size and number.

The objectives of this study were to (a) estimate GCA, SCA, and reciprocal effects for yield components of selected snap bean parents and progeny under high and low temperature stress, and (b) investigate potential association between high and low temperature stress tolerances during reproductive development in snap bean. 


\section{Materials and methods}

Ten snap bean breeding lines and cultivars comprised of one chilling tolerant inbred breeding line ('CT 70', Cornell breeding line), two heat sensitive cultivars ('Hystyle', Harris Moran; 'Labrador', Seminis) and seven heat tolerant inbred lines and cultivars ('Barrier', Alpha Seeds; 'Brio', Seminis; 'Carson' [wax bean], Syngenta; 'Cornell 502', Cornell breeding line; 'HB1880', Syngenta; 'Opus', Seminis; 'Venture', Syngenta). The snap bean parents used were all large sieve size (4-5), determinate type I growth habit, self-pollinated lines. Parents were crossed in all possible combinations using the hooking method without emasculation (Bliss, 1980). A complete set of 90 $F_{1}$ 's (45 hybrids with 45 reciprocals) was created for each temperature treatment. A sample of 100 progeny assayed with RAPD markers (Williams et al., 1990) unique to each parent (University of British Columbia primers, Vancouver, B.C., Canada) indicated that the percentage of selfs was close to zero (data not shown).

The cold test was planted April 12, 2003 and the heat test was planted June 5, 2003. For each temperature treatment, the $90 F_{1}$ 's and 10 parents were planted and randomized in four replications divided between two identical $90 \mathrm{~m}^{2}$ greenhouses. To account for reciprocal/maternal effects among $F_{1}$ 's, reciprocal crosses in one direction were planted in two of the replications, and crosses in the other direction were planted in the other two replications. Seeds of $F_{1}$ hybrids and parents were sown in $14.6 \mathrm{~cm} \times 14.6 \mathrm{~cm}$ square pots filled with 'Cornell mix' (Boodley \& Sheldrake, 1972). Greenhouses were set for a $14 \mathrm{hr}$ photoperiod with supplemental lighting provided by $1000 \mathrm{~W}$ metal halide bulbs (SunSystem III from Sunlight Supply, Inc., Vancouver, WA) at a rate of $300 \mu \mathrm{M} \mathrm{m}^{-2} \mathrm{~s}^{-1}$ of photosynthetically active radiation at bench level. Greenhouse temperatures were set at $24{ }^{\circ} \mathrm{C}$ day $/ 21^{\circ} \mathrm{C}$ night for germination and development of seedlings.

Temperature treatments were imposed two weeks after seedling emergence, corresponding to microsporogenesis, and continued through plant senescence. Low temperature treatment was $16^{\circ} \mathrm{C}$ day $/ 10^{\circ} \mathrm{C}$ night, high temperature treatment was $32{ }^{\circ} \mathrm{C}$ day $/ 28^{\circ} \mathrm{C}$ night. Plants were irrigated as needed and fertilized equally. A guard row surrounded each experiment to maintain uniform light and temperature distribution, microclimate humidity, and air movement. After complete plant senescence all pods were harvested from individual plants. Pod and seed counts, and seed weight (to $1 \times 10^{-2} \mathrm{~g}$ ) were recorded separately for each plant in the experiment.

Yield component data were analyzed using the general linear model (GLM) analysis, where type III sums of squares comparisons were used to obtain $F$ values for experimental variables, and comparisons of means of yield components were obtained using Duncan's multiple range test $(P \leq 0.05)$ (SAS, 1997). GLM analysis indicated reciprocal effects were not significant, so reciprocal values were used for missing crosses in the diallel analysis (only one hybrid in one direction, 'Venture' $\times$ 'Labrador' [female $\times$ male], did not germinate in any case). Means for treatment replications were used to calculate GCA, SCA, reciprocal, and maternal effects with the program DIALLELSAS (Zhang \& Kang, 1997), based on Griffing's model I, method I diallel analysis (fixed effects) (Griffing, 1956). Correlations among yield component means and GCA values within separate temperature treatments were calculated using Pearson product-moment correlations (SAS, 1997). Correlations among yield components and GCA values across temperature treatments were calculated using Spearman's rank-correlation coefficient (SAS, 1997).

\section{Results and discussion}

Significant variation for yield components (YC) was observed among parents and $F_{1}$ 's at high and low temperatures (Tables 1 and 2). High temperature treatment of the $F_{1}$ 's and parents resulted in significantly lower $(P \leq 0.01)$ means for all YC as compared to the low temperature treatment (data not shown). Analysis of variance indicated significant $(P \leq 0.0001)$ greenhouse and replication effects in the heat treatment, and significant $(P \leq 0.0001)$ replication effects in the chilling treatment (data not shown). Yield component means under high and low temperature treatment, respectively, were 10.04 and 10.76 for pod number, 27.23 and 33.38 for seed number, 4.46 and $10.23 \mathrm{~g}$ for seed weight, 0.162 and $0.317 \mathrm{~g}$ for mean seed weight, and 2.56 and 3.10 for seeds per pod. Pod number, seed number, and seeds per pod were the best indicators of yield in this study. Reciprocal effects were not significant for any full-sib family (i.e. cross) (data not shown), or YC except seed number in high temperature at $P \leq 0.05$ (Table 3).

Variation in GCA was highly significant $(P \leq 0.0001)$ for pod number, seed number, and seeds per pod in both temperature treatments (Table 3). 
Table 1. Mean pod number, mean seed number, and mean seeds per pod for the 10 parents and 45 hybrids of a diallel analysis when grown in a high temperature $\left(32^{\circ} \mathrm{C}\right.$ day $/ 28^{\circ} \mathrm{C}$ night $)$ controlled environment

\begin{tabular}{|c|c|c|c|c|c|c|c|c|}
\hline \multirow[b]{2}{*}{ Rank } & \multirow[b]{2}{*}{ Hybrid or parent } & \multicolumn{2}{|c|}{ Pods (no.) } & \multicolumn{2}{|c|}{ Seeds (no.) } & \multicolumn{2}{|c|}{ Seeds (no./pod) } & \multirow[b]{2}{*}{ Mean rank } \\
\hline & & Value & YC rank & Value & YC rank & Value & YC rank & \\
\hline 1 & $\mathrm{Car} \times \mathrm{CT} 70$ & $12.5 \mathrm{a}-\mathrm{c}$ & 2 & $45.3 \mathrm{a}$ & 1 & $3.65 \mathrm{a}$ & 2 & 1.7 \\
\hline 2 & C $502 \times \mathrm{HB}$ & $11.89 \mathrm{a}-\mathrm{e}$ & 5 & $42.56 \mathrm{a}-\mathrm{c}$ & 3 & $3.55 \mathrm{ab}$ & 3 & 3.7 \\
\hline 3 & Brio $\times$ C 502 & $13.6 \mathrm{a}$ & 1 & $43.9 \mathrm{ab}$ & 2 & $3.21 \mathrm{a}-\mathrm{g}$ & 10 & 4.3 \\
\hline 4 & CT $70 \times \mathrm{HB}$ & $11.89 \mathrm{a}-\mathrm{e}$ & 6 & $42.56 \mathrm{a}-\mathrm{c}$ & 4 & $3.55 \mathrm{ab}$ & 4 & 4.7 \\
\hline 5 & Brio $\times$ Ven & $11.7 \mathrm{a}-\mathrm{e}$ & 8 & $40.7 \mathrm{a}-\mathrm{e}$ & 5 & $3.39 \mathrm{a}-\mathrm{d}$ & 7 & 6.7 \\
\hline 6 & C $502 \times$ Ven & $11.45 \mathrm{a}-\mathrm{e}$ & 13 & $38.35 \mathrm{a}-\mathrm{f}$ & 6 & $3.45 \mathrm{a}-\mathrm{c}$ & 5 & 8.0 \\
\hline 7 & CT $70 \times$ Ven & $11.45 \mathrm{a}-\mathrm{e}$ & 14 & $38.35 \mathrm{a}-\mathrm{f}$ & 7 & $3.45 \mathrm{a}-\mathrm{c}$ & 6 & 9.0 \\
\hline 8 & Brio $\times \mathrm{HB}$ & $12.2 \mathrm{a}-\mathrm{d}$ & 3 & $36.95 \mathrm{~b}-\mathrm{g}$ & 8 & $2.99 \mathrm{~b}-\mathrm{k}$ & 16 & 9.0 \\
\hline 9 & Brio $\times$ CT 70 & $11.6 \mathrm{a}-\mathrm{e}$ & 9 & $35.55 \mathrm{c}-\mathrm{i}$ & 12 & $3.08 \mathrm{a}-\mathrm{j}$ & 15 & 12.0 \\
\hline 10 & Bar × C 502 & $11.75 \mathrm{a}-\mathrm{e}$ & 7 & $34.7 \mathrm{~d}-\mathrm{j}$ & 14 & $2.85 \mathrm{c}-1$ & 22 & 14.3 \\
\hline 11 & C $502 \times$ Lab & $10.9 \mathrm{a}-\mathrm{g}$ & 24 & $35.9 \mathrm{c}-\mathrm{h}$ & 9 & $3.18 \mathrm{a}-\mathrm{h}$ & 12 & 15.0 \\
\hline 12 & CT $70 \times$ Lab & $10.9 \mathrm{a}-\mathrm{g}$ & 25 & $35.9 \mathrm{c}-\mathrm{h}$ & 10 & $3.18 \mathrm{a}-\mathrm{h}$ & 13 & 16.0 \\
\hline 13 & Bar $\times$ Ven & $11.45 \mathrm{a}-\mathrm{e}$ & 12 & $34.15 \mathrm{~d}-\mathrm{k}$ & 15 & $2.92 \mathrm{~b}-1$ & 21 & 16.0 \\
\hline 14 & $\mathrm{HB} \times \mathrm{Ven}$ & $9.74 \mathrm{~d}-\mathrm{i}$ & 37 & $35.63 \mathrm{c}-\mathrm{i}$ & 11 & $3.70 \mathrm{a}$ & 1 & 16.3 \\
\hline 15 & Car $\times$ Ven & $10.53 \mathrm{~b}-\mathrm{h}$ & 28 & $34.74 \mathrm{~d}-\mathrm{j}$ & 13 & $3.31 \mathrm{a}-\mathrm{e}$ & 8 & 16.3 \\
\hline 16 & C $502 \times$ Opus & $11.2 \mathrm{a}-\mathrm{g}$ & 19 & $33.9 \mathrm{~d}-1$ & 16 & $2.99 \mathrm{~b}-\mathrm{k}$ & 17 & 17.3 \\
\hline 17 & CT $70 \times$ Opus & $11.2 \mathrm{a}-\mathrm{g}$ & 20 & $33.9 \mathrm{~d}-1$ & 17 & $2.99 \mathrm{~b}-\mathrm{k}$ & 18 & 18.3 \\
\hline 18 & $\mathrm{Car} \times \mathrm{Lab}$ & $11.95 \mathrm{a}-\mathrm{d}$ & 4 & $31.1 \mathrm{f}-\mathrm{o}$ & 24 & $2.57 \mathrm{~h}-\mathrm{p}$ & 30 & 19.3 \\
\hline 19 & C $502 \times$ CT 70 & $11.3 \mathrm{a}-\mathrm{f}$ & 16 & $33.65 \mathrm{e}-\mathrm{m}$ & 18 & $2.79 \mathrm{~d}-\mathrm{m}$ & 26 & 20.0 \\
\hline 20 & $\mathrm{Car} \times \mathrm{HB}$ & $10.2 \mathrm{~b}-\mathrm{h}$ & 32 & $32.05 \mathrm{f}-\mathrm{n}$ & 21 & $3.23 \mathrm{a}-\mathrm{f}$ & 9 & 20.7 \\
\hline 21 & Lab $\times$ Ven & $11.5 \mathrm{a}-\mathrm{e}$ & 11 & $30.1 \mathrm{~g}-\mathrm{p}$ & 25 & $2.66 \mathrm{~g}-\mathrm{p}$ & 28 & 21.3 \\
\hline 22 & Brio $\times$ Car & $11.2 \mathrm{a}-\mathrm{f}$ & 18 & $31.5 \mathrm{f}-\mathrm{n}$ & 23 & $2.8 \mathrm{~d}-\mathrm{n}$ & 25 & 22.0 \\
\hline 23 & Car $\times$ Hys & $10.1 \mathrm{~b}-\mathrm{h}$ & 33 & $32.3 \mathrm{f}-\mathrm{m}$ & 20 & $3.16 \mathrm{a}-\mathrm{i}$ & 14 & 22.3 \\
\hline 24 & $\mathrm{Car} \times \mathrm{C} 5$ & $10.5 \mathrm{c}-\mathrm{h}$ & 29 & $33.2 \mathrm{e}-\mathrm{m}$ & 19 & $2.99 \mathrm{~b}-\mathrm{k}$ & 19 & 22.3 \\
\hline 25 & Car $\times$ Opus & $10 \mathrm{~b}-\mathrm{i}$ & 35 & $31.75 \mathrm{f}-\mathrm{n}$ & 22 & $3.21 \mathrm{a}-\mathrm{g}$ & 11 & 22.7 \\
\hline 26 & Opus $\times$ Ven & $11.3 \mathrm{a}-\mathrm{f}$ & 17 & $27.7 \mathrm{i}-\mathrm{r}$ & 28 & $2.421-\mathrm{s}$ & 33 & 26.0 \\
\hline 27 & Hys $\times$ Ven & $10.05 \mathrm{~b}-\mathrm{h}$ & 34 & $29 \mathrm{~g}-\mathrm{q}$ & 26 & $2.95 \mathrm{~b}-1$ & 20 & 26.7 \\
\hline 28 & Bar $\times$ Brio & $10.25 \mathrm{~b}-\mathrm{h}$ & 30 & $28.6 \mathrm{~h}-\mathrm{r}$ & 27 & $2.74 \mathrm{e}-\mathrm{O}$ & 27 & 28.0 \\
\hline 29 & Brio $\times$ Hys & $10.9 \mathrm{a}-\mathrm{g}$ & 22 & $27.2 \mathrm{j}-\mathrm{s}$ & 30 & $2.47 \mathrm{k}-\mathrm{q}$ & 32 & 28.0 \\
\hline 30 & $\mathrm{Bar} \times \mathrm{Car}$ & $10.65 \mathrm{~b}-\mathrm{h}$ & 27 & $27.55 \mathrm{~h}-\mathrm{r}$ & 29 & $2.59 \mathrm{~g}-\mathrm{q}$ & 29 & 28.3 \\
\hline 31 & $\mathrm{Bar} \times \mathrm{Lab}$ & $11.35 \mathrm{a}-\mathrm{f}$ & 15 & $26.051-\mathrm{t}$ & 33 & $2.311-\mathrm{u}$ & 37 & 28.3 \\
\hline 32 & Hys $\times$ Opus & $11.6 \mathrm{a}-\mathrm{e}$ & 10 & $24.07 \mathrm{n}-\mathrm{u}$ & 36 & $2.07 \mathrm{p}-\mathrm{v}$ & 43 & 29.7 \\
\hline 33 & C $502 \times$ Hys & $9.32 \mathrm{e}-\mathrm{j}$ & 39 & $26.9 \mathrm{j}-\mathrm{s}$ & 31 & $2.83 \mathrm{c}-\mathrm{m}$ & 23 & 31.0 \\
\hline 34 & Lab $\times$ Opus & $10.89 \mathrm{a}-\mathrm{g}$ & 26 & $25.941-\mathrm{t}$ & 34 & $2.41 \mathrm{k}-\mathrm{s}$ & 35 & 31.7 \\
\hline 35 & CT $70 \times$ Hys & $9.32 \mathrm{e}-\mathrm{j}$ & 40 & $26.9 \mathrm{j}-\mathrm{s}$ & 32 & $2.83 \mathrm{c}-\mathrm{m}$ & 24 & 32.0 \\
\hline 36 & Bar $\times$ Hys & $10.25 \mathrm{~b}-\mathrm{h}$ & 31 & $25.69 \mathrm{~m}-\mathrm{t}$ & 35 & $2.421-\mathrm{s}$ & 34 & 33.3 \\
\hline 37 & Brio $\times$ Lab & $10.9 \mathrm{a}-\mathrm{g}$ & 23 & $23.35 \mathrm{o}-\mathrm{v}$ & 37 & $2.15 \mathrm{o}-\mathrm{V}$ & 41 & 33.7 \\
\hline 38 & $\mathrm{HB} \times$ Opus & $11.2 \mathrm{a}-\mathrm{g}$ & 21 & $22.13 \mathrm{p}-\mathrm{v}$ & 38 & $2.01 \mathrm{q}-\mathrm{w}$ & 45 & 34.7 \\
\hline 39 & Carson & $9.55 \mathrm{~d}-\mathrm{i}$ & 38 & $21.05 \mathrm{q}-\mathrm{v}$ & 40 & $2.19 \mathrm{n}-\mathrm{v}$ & 39 & 39.0 \\
\hline 40 & Bar $\times$ CT 70 & $7.43 \mathrm{i}-1$ & 48 & $21.43 \mathrm{q}-\mathrm{v}$ & 39 & $2.53 \mathrm{i}-\mathrm{q}$ & 31 & 39.33 \\
\hline 41 & Bar × HB & $8.55 \mathrm{~g}-\mathrm{k}$ & 45 & 20.9 r-w & 41 & $2.371-\mathrm{r}$ & 36 & 40.7 \\
\hline 42 & HB 1880 & $8.65 \mathrm{f}-\mathrm{j}$ & 44 & $20 s-w$ & 43 & $2.21 \mathrm{~m}-\mathrm{v}$ & 38 & 41.7 \\
\hline 43 & Brio $\times$ Opus & $8.65 \mathrm{f}-\mathrm{k}$ & 43 & $20.2 \mathrm{~s}-\mathrm{W}$ & 42 & $2.07 \mathrm{p}-\mathrm{v}$ & 44 & 43.0 \\
\hline
\end{tabular}


Table 1. (Continued)

\begin{tabular}{|c|c|c|c|c|c|c|c|c|}
\hline \multirow[b]{2}{*}{ Rank } & \multirow[b]{2}{*}{ Hybrid or parent } & \multicolumn{2}{|c|}{ Pods (no.) } & \multicolumn{2}{|c|}{ Seeds (no.) } & \multicolumn{2}{|c|}{ Seeds (no./pod) } & \multirow[b]{2}{*}{ Mean rank } \\
\hline & & Value & YC rank & Value & YC rank & Value & YC rank & \\
\hline 44 & $\mathrm{HB} \times \mathrm{Lab}$ & $9.8 \mathrm{~d}-\mathrm{i}$ & 36 & $18.8 \mathrm{t}-\mathrm{x}$ & 44 & $1.76 \mathrm{t}-\mathrm{x}$ & 49 & 43.0 \\
\hline 45 & Brio & $8.05 \mathrm{~h}-1$ & 47 & $16.65 \mathrm{u}-\mathrm{x}$ & 45 & $2.15 \mathrm{q}-\mathrm{o}$ & 42 & 44.7 \\
\hline 46 & Bar $\times$ Opus & $6.95 \mathrm{j}-\mathrm{m}$ & 49 & $16.6 \mathrm{u}-\mathrm{y}$ & 46 & $2.18 \mathrm{n}-\mathrm{v}$ & 40 & 45.0 \\
\hline 47 & Hys $\times$ Lab & $8.2 \mathrm{~h}-\mathrm{k}$ & 46 & $16.55 \mathrm{u}-\mathrm{y}$ & 47 & $1.98 \mathrm{q}-\mathrm{w}$ & 46 & 46.3 \\
\hline 48 & $\mathrm{HB} \times$ Hys & $9.27 \mathrm{e}-\mathrm{j}$ & 42 & $16 v-y$ & 48 & $1.74 \mathrm{u}-\mathrm{x}$ & 50 & 46.7 \\
\hline 49 & Cornell 502 & $9.3 \mathrm{e}-\mathrm{j}$ & 41 & $15.6 \mathrm{v}-\mathrm{y}$ & 49 & $1.59 \mathrm{v}-\mathrm{x}$ & 51 & 47.0 \\
\hline 50 & Opus & $6.42 \mathrm{k}-\mathrm{m}$ & 50 & $13.16 \mathrm{w}-\mathrm{z}$ & 50 & $1.87 \mathrm{r}-\mathrm{x}$ & 47 & 49.0 \\
\hline 51 & CT 70 & $6.05 \mathrm{~lm}$ & 51 & $11.95 \mathrm{x}-\mathrm{Z}$ & 51 & $1.82 \mathrm{~s}-\mathrm{x}$ & 48 & 50.0 \\
\hline 52 & Barrier & $5.37 \mathrm{~m}$ & 54 & $10.05 \mathrm{yz}$ & 52 & $1.46 \mathrm{w}-\mathrm{y}$ & 52 & 52.7 \\
\hline 53 & Venture & $5.95 \mathrm{~lm}$ & 52 & $9.3 \mathrm{yz}$ & 53 & 1.33 xy & 53 & 52.7 \\
\hline 54 & Hystyle & $5.42 \mathrm{~m}$ & 53 & $6.16 \mathrm{z}-\mathrm{aa}$ & 54 & $1.02 \mathrm{yz}$ & 54 & 53.7 \\
\hline 55 & $\begin{array}{l}\text { Labrador } \\
\text { S.D. }\end{array}$ & $\begin{aligned} & 2.95 \mathrm{n} \\
\pm & 3.39\end{aligned}$ & 55 & $\begin{array}{r}2.2 \mathrm{aa} \\
\pm 11.78\end{array}$ & 55 & $\begin{array}{l}0.54 \mathrm{z} \\
\pm 0.78\end{array}$ & 55 & 55.0 \\
\hline
\end{tabular}

Hybrids and parents are arranged according to mean rank. Overall rank in the first column of hybrid or parent is based on mean rank. Mean rank is the mean of the three YC ranks. YC rank: yield component rank (rank of hybrid or parent for pod number, seed number, or seeds per pod); Car: 'Carson'; C 502: 'Cornell 502'; HB: 'HB 1880'; Ven: 'Venture'; Lab: 'Labrador'; Hys: 'Hystyle'. Within each yield component the means followed by different letters are significantly different $(P \leq 0.01)$ according to Duncan's multiple range test.

The observation of significant GCA in each treatment indicated additive genetic variance contributed to heat tolerance in the parental population. However, additive genetic variance may be overestimated in a diallel analysis of non-randomly selected parents. The predominance of GCA contribution to genotypic variance for seed number is typical in common bean (Dickson, 1967; Kornegay et al., 1992; Nienhuis \& Singh, 1988; Porch, 2001; Singh et al., 1992), and significant positive GCA for seed number in common bean has been observed previously in populations from diverse gene pools and races (Abreau et al., 1999; Nienhuis \& Singh, 1988; Singh et al., 1992). Large additive effects also have been reported for pod number (Dickson, 1967; Nienhuis \& Singh, 1988; Porch, 2001) and seeds per pod (Dickson, 1967; Mumba \& Galwey, 1999; Nienhuis \& Singh, 1988; Porch, 2001).

Significant $(P \leq 0.05)$ SCA variance was observed in both temperature treatments (Table 3), indicating non-additive genetic contribution to yield as well. The statistical significance of the components of genetic variance indicated that genetic variation for pod number, seed number, and seeds per pod under both temperature treatments was present in the diallel mating design, and that experimental conditions allowed for the detection of genetic differences among the parents and progeny. SCA variance is unlikely to be overestimated in this study as analysis of variance indicated heterosis was insignificant within families. Observation of non-additive gene action under temperature stress due to significant SCA contribution to variance provides further evidence that evaluation beyond the F1 generation may be necessary. This result differs from previous studies in which heterosis for yield and yield components was found to be significant in dry bean (Foolad \& Bassiri, 1983; Nienhuis \& Singh, 1986; Shonnard \& Gepts, 1994). The lack of heterosis in this study may be attributed to uniformity of parents used in this experiment, as high $F_{1}$ heterosis in bean is associated with increasing phenotypic and genotypic divergence between parents (Evans, 1970; Nienhuis \& Singh, 1986). Observation of insignificant heterosis suggests SCA can be utilized in selecting the best parental combinations for developing heat tolerant snap beans.

A number of interactions occurred between genotype and temperature. Parental performance suggested different alleles, genes, and combinations of genes may be available for breeding improved cultivars. For example, 'Cornell 502', was heat tolerant and chilling sensitive; thus, the gene or genes influencing yield under high temperature in 'Cornell 502' did not contribute to cold tolerance. Conversely, the cultivar 'Labrador' was chilling tolerant and heat sensitive. Parental performance under high and low temperatures was associated 
Table 2. Mean pod number, mean seed number, and mean seeds per pod for the 10 parents and 45 hybrids of a diallel analysis when grown in a low temperature $\left(16^{\circ} \mathrm{C}\right.$ day $/ 10^{\circ} \mathrm{C}$ night $)$ controlled environment

\begin{tabular}{|c|c|c|c|c|c|c|c|c|}
\hline \multirow[b]{2}{*}{ Rank } & \multirow[b]{2}{*}{ Hybrid or parent } & \multicolumn{2}{|c|}{ Pods (no.) } & \multicolumn{2}{|c|}{ Seeds (no.) } & \multicolumn{2}{|c|}{ Seeds (no./pod) } & \multirow[b]{2}{*}{ Mean rank } \\
\hline & & Value & YC rank & Value & YC rank & Value & YC rank & \\
\hline 1 & Brio & $13.7 \mathrm{ab}$ & 2 & $50 \mathrm{a}$ & 1 & $3.67 \mathrm{a}-\mathrm{h}$ & 11 & 4.7 \\
\hline 2 & Brio $\times$ CT 70 & $12.84 \mathrm{a}-\mathrm{f}$ & 7 & $43.79 \mathrm{a}-\mathrm{d}$ & 4 & $3.7 \mathrm{a}-\mathrm{h}$ & 10 & 7.0 \\
\hline 3 & Brio $\times \mathrm{HB}$ & $12.88 \mathrm{a}-\mathrm{e}$ & 6 & $46.53 \mathrm{ab}$ & 2 & $3.63 \mathrm{a}-\mathrm{i}$ & 15 & 7.7 \\
\hline 4 & Brio $\times$ Lab & $12.75 \mathrm{a}-\mathrm{g}$ & 8 & $44.35 \mathrm{a}-\mathrm{c}$ & 3 & $3.44 \mathrm{~b}-1$ & 21 & 10.7 \\
\hline 5 & Brio $\times$ Ven & $10.76 c-n$ & 27 & $41.53 \mathrm{~b}-\mathrm{f}$ & 6 & $3.92 \mathrm{ab}$ & 2 & 11.7 \\
\hline 6 & Brio $\times$ C 502 & $12.35 \mathrm{~b}-\mathrm{h}$ & 11 & $43.2 \mathrm{a}-\mathrm{e}$ & 5 & $3.52 \mathrm{~b}-\mathrm{k}$ & 20 & 12.0 \\
\hline 7 & Bar $\times$ Lab & $13.11 \mathrm{a}-\mathrm{d}$ & 4 & $41.39 \mathrm{~b}-\mathrm{f}$ & 7 & $3.23 \mathrm{e}-\mathrm{q}$ & 26 & 12.3 \\
\hline 8 & Bar $\times$ Ven & $12.2 \mathrm{~b}-\mathrm{i}$ & 14 & $41.25 \mathrm{~b}-\mathrm{f}$ & 8 & $3.38 \mathrm{~b}-\mathrm{m}$ & 22 & 14.7 \\
\hline 9 & $\mathrm{Lab} \times \mathrm{Ven}$ & $10 \mathrm{~g}-\mathrm{n}$ & 38 & $41 \mathrm{~b}-\mathrm{f}$ & 9 & $4.1 \mathrm{a}$ & 1 & 16.0 \\
\hline 10 & Brio $\times$ Car & $11.8 \mathrm{~b}-\mathrm{j}$ & 16 & $39.45 \mathrm{~b}-\mathrm{g}$ & 10 & $3.35 \mathrm{~b}-\mathrm{n}$ & 23 & 16.3 \\
\hline 11 & Car $\times$ Lab & $10.21 \mathrm{e}-\mathrm{n}$ & 32 & $38.68 \mathrm{~b}-\mathrm{h}$ & 14 & $3.82 \mathrm{a}-\mathrm{d}$ & 5 & 17.0 \\
\hline 12 & $\mathrm{HB} \times$ Ven & $10.43 \mathrm{~d}-\mathrm{n}$ & 30 & $38.71 \mathrm{~b}-\mathrm{h}$ & 13 & $3.79 \mathrm{a}-\mathrm{f}$ & 8 & 17.0 \\
\hline 13 & $\mathrm{HB} \times \mathrm{Lab}$ & $10.82 c-n$ & 24 & $38.77 \mathrm{~b}-\mathrm{h}$ & 12 & $3.61 \mathrm{a}-\mathrm{j}$ & 16 & 17.3 \\
\hline 14 & Car $\times$ Opus & $12.3 \mathrm{~b}-\mathrm{i}$ & 13 & $38.95 \mathrm{~b}-\mathrm{h}$ & 11 & $3.18 \mathrm{~g}-\mathrm{q}$ & 34 & 19.3 \\
\hline 15 & $\mathrm{Car} \times \mathrm{HB}$ & $10.78 c-n$ & 26 & $38.56 \mathrm{~b}-\mathrm{i}$ & 15 & $3.57 \mathrm{a}-\mathrm{j}$ & 18 & 19.7 \\
\hline 16 & Brio $\times$ Opus & $11.94 \mathrm{~b}-\mathrm{j}$ & 15 & $37.61 \mathrm{e}-\mathrm{i}$ & 17 & $3.23 \mathrm{e}-\mathrm{q}$ & 27 & 19.7 \\
\hline 17 & Opus $\times$ Ven & $11.3 \mathrm{~b}-\mathrm{n}$ & 21 & $37.55 \mathrm{c}-\mathrm{i}$ & 18 & $3.33 \mathrm{c}-\mathrm{o}$ & 24 & 21.0 \\
\hline 18 & Bar × CT 70 & $12.33 \mathrm{~d}-\mathrm{h}$ & 12 & $38.5 \mathrm{~b}-\mathrm{i}$ & 16 & $3.16 \mathrm{~h}-\mathrm{q}$ & 35 & 21.0 \\
\hline 19 & C $502 \times$ Opus & $11.5 \mathrm{~b}-\mathrm{m}$ & 18 & $36.67 \mathrm{c}-\mathrm{j}$ & 20 & $3.21 \mathrm{~h}-\mathrm{q}$ & 29 & 22.3 \\
\hline 20 & $\mathrm{Bar} \times \mathrm{Car}$ & $13.05 \mathrm{a}-\mathrm{d}$ & 5 & $37.47 \mathrm{~d}-\mathrm{i}$ & 19 & $2.85 \mathrm{~m}-\mathrm{t}$ & 43 & 22.3 \\
\hline 21 & C $502 \times$ Lab & $9.5 \mathrm{i}-\mathrm{o}$ & 43 & $35.58 \mathrm{~d}-1$ & 24 & $3.85 \mathrm{a}-\mathrm{c}$ & 3 & 23.3 \\
\hline 22 & CT $70 \times$ Opus & $11.5 \mathrm{~b}-\mathrm{m}$ & 19 & $36.67 \mathrm{c}-\mathrm{j}$ & 21 & $3.21 \mathrm{~h}-\mathrm{q}$ & 30 & 23.3 \\
\hline 23 & Brio $\times$ Hys & $11.25 \mathrm{~b}-\mathrm{n}$ & 22 & $36.2 \mathrm{c}-\mathrm{k}$ & 22 & $3.22 \mathrm{~g}-\mathrm{q}$ & 28 & 24.0 \\
\hline 24 & CT $70 \times$ Lab & $9.5 \mathrm{i}-\mathrm{o}$ & 44 & $35.58 \mathrm{~d}-1$ & 25 & $3.85 \mathrm{a}-\mathrm{c}$ & 4 & 24.3 \\
\hline 25 & $\mathrm{Car} \times \mathrm{CT} 70$ & $9.6 \mathrm{~h}-\mathrm{n}$ & 41 & $35.55 \mathrm{~d}-1$ & 26 & $3.74 \mathrm{a}-\mathrm{g}$ & 9 & 25.3 \\
\hline 26 & $\mathrm{HB} \times$ Opus & $13.31 \mathrm{a}-\mathrm{c}$ & 3 & $35.31 \mathrm{~d}-1$ & 27 & $2.68 \mathrm{q}-\mathrm{u}$ & 46 & 25.3 \\
\hline 27 & Car $\times$ Ven & $10.05 \mathrm{f}-\mathrm{n}$ & 37 & $35.8 \mathrm{c}-1$ & 23 & $3.6 \mathrm{a}-\mathrm{j}$ & 17 & 25.7 \\
\hline 28 & C $502 \times \mathrm{HB}$ & $9.61 \mathrm{~h}-\mathrm{o}$ & 39 & $34.83 \mathrm{e}-\mathrm{m}$ & 30 & $3.64 \mathrm{a}-\mathrm{i}$ & 13 & 27.3 \\
\hline 29 & Bar $\times$ Brio & $12.5 \mathrm{~b}-\mathrm{g}$ & 9 & $35 \mathrm{f}-\mathrm{m}$ & 28 & $2.66 \mathrm{q}-\mathrm{u}$ & 47 & 28.0 \\
\hline 30 & $\mathrm{CT} 70 \times \mathrm{HB}$ & $9.61 \mathrm{~h}-\mathrm{o}$ & 40 & $34.83 \mathrm{e}-\mathrm{m}$ & 31 & $3.64 \mathrm{a}-\mathrm{i}$ & 14 & 28.3 \\
\hline 31 & Labrador & $9.35 \mathrm{j}-\mathrm{o}$ & 45 & $34.95 \mathrm{e}-\mathrm{m}$ & 29 & $3.65 \mathrm{a}-\mathrm{i}$ & 12 & 28.7 \\
\hline 32 & C $502 \times$ Ven & $8.891-\mathrm{o}$ & 49 & $33.16 \mathrm{f}-\mathrm{n}$ & 33 & $3.81 \mathrm{a}-\mathrm{e}$ & 6 & 29.3 \\
\hline 33 & Bar $\times$ Opus & $15.15 \mathrm{a}$ & 1 & $31.55 \mathrm{~g}-\mathrm{n}$ & 37 & $2.08 \mathrm{vw}$ & 52 & 30.0 \\
\hline 34 & CT $70 \times$ Ven & $8.891-\mathrm{o}$ & 50 & $33.16 \mathrm{f}-\mathrm{n}$ & 34 & $3.81 \mathrm{a}-\mathrm{e}$ & 7 & 30.3 \\
\hline 35 & Lab $\times$ Opus & $10.75 \mathrm{c}-\mathrm{n}$ & 28 & $34.5 \mathrm{e}-\mathrm{n}$ & 32 & $3.2 \mathrm{~h}-\mathrm{q}$ & 31 & 30.3 \\
\hline 36 & $\mathrm{Bar} \times \mathrm{HB}$ & $12.47 \mathrm{~b}-\mathrm{g}$ & 10 & $31.47 \mathrm{~h}-\mathrm{n}$ & 39 & $2.54 \mathrm{r}-\mathrm{v}$ & 48 & 32.3 \\
\hline 37 & C $502 \times$ Hys & 10.17 e-n & 33 & $31.67 \mathrm{~h}-\mathrm{n}$ & 35 & $3.2 \mathrm{f}-\mathrm{q}$ & 32 & 33.3 \\
\hline 38 & CT $70 \times$ Hys & $10.17 \mathrm{e}-\mathrm{n}$ & 34 & $31.67 \mathrm{~h}-\mathrm{n}$ & 36 & $3.2 \mathrm{f}-\mathrm{q}$ & 33 & 34.3 \\
\hline 39 & Hys $\times$ Lab & $10.46 \mathrm{~d}-\mathrm{n}$ & 29 & $31.54 \mathrm{~b}-\mathrm{h}$ & 38 & $3.02 \mathrm{j}-\mathrm{t}$ & 37 & 34.7 \\
\hline 40 & $\mathrm{Car} \times \mathrm{C} 5$ & $10.8 \mathrm{c}-\mathrm{n}$ & 25 & $30.75 \mathrm{~g}-\mathrm{o}$ & 40 & $2.861-\mathrm{t}$ & 42 & 35.7 \\
\hline 41 & Hys $\times$ Opus & $11.56 \mathrm{~b}-1$ & 17 & $29.75 \mathrm{i}-\mathrm{p}$ & 42 & $2.53 \mathrm{~s}-\mathrm{v}$ & 49 & 36.0 \\
\hline 42 & Hys $\times$ Ven & 8.62 on & 52 & $30.69 \mathrm{~g}-\mathrm{o}$ & 41 & $3.55 \mathrm{a}-\mathrm{k}$ & 19 & 37.3 \\
\hline 43 & Bar $\times$ C 502 & $10.35 \mathrm{~d}-\mathrm{n}$ & 31 & $28.25 \mathrm{j}-\mathrm{q}$ & 43 & $2.76 \mathrm{o}-\mathrm{u}$ & 44 & 39.3 \\
\hline 44 & Bar $\times$ Hys & $11.21 \mathrm{~b}-\mathrm{n}$ & 23 & $28 \mathrm{j}-\mathrm{q}$ & 44 & $2.27 \mathrm{u}-\mathrm{w}$ & 51 & 39.3 \\
\hline
\end{tabular}


Table 2. (Continued)

\begin{tabular}{|c|c|c|c|c|c|c|c|c|}
\hline \multirow[b]{2}{*}{ Rank } & \multirow[b]{2}{*}{ Hybrid or parent } & \multicolumn{2}{|c|}{ Pods (no.) } & \multicolumn{2}{|c|}{ Seeds (no.) } & \multicolumn{2}{|c|}{ Seeds (no./pod) } & \multirow[b]{2}{*}{ Mean rank } \\
\hline & & Value & YC rank & Value & YC rank & Value & YC rank & \\
\hline 45 & CT 70 & $8.7 \mathrm{~m}-\mathrm{o}$ & 51 & $27.75 \mathrm{k}-\mathrm{q}$ & 46 & $3.26 \mathrm{~d}-\mathrm{p}$ & 25 & 40.7 \\
\hline 46 & Barrier & $11.35 \mathrm{~b}-\mathrm{n}$ & 20 & $22.7 \mathrm{o}-\mathrm{r}$ & 51 & $1.95 \mathrm{wx}$ & 53 & 41.3 \\
\hline 47 & $\mathrm{HB} \times \mathrm{Hys}$ & $9.57 \mathrm{~h}-\mathrm{n}$ & 42 & $27.79 \mathrm{k}-\mathrm{q}$ & 45 & $2.91-\mathrm{t}$ & 41 & 42.7 \\
\hline 48 & HB 1880 & $10.16 \mathrm{f}-\mathrm{n}$ & 35 & $27.051-\mathrm{q}$ & 49 & $2.73 \mathrm{q}-\mathrm{u}$ & 45 & 43.0 \\
\hline 49 & Car $\times$ Hys & $8.94 \mathrm{k}-\mathrm{O}$ & 48 & $27.47 \mathrm{k}-\mathrm{q}$ & 48 & $3.08 \mathrm{i}-\mathrm{r}$ & 36 & 44.0 \\
\hline 50 & C $502 \times$ CT 70 & $9.3 \mathrm{j}-\mathrm{o}$ & 46 & $27.6 \mathrm{k}-\mathrm{q}$ & 47 & $2.931-\mathrm{t}$ & 40 & 44.3 \\
\hline 51 & Carson & $8.95 \mathrm{k}-\mathrm{o}$ & 47 & $26.4 \mathrm{~m}-\mathrm{r}$ & 50 & $2.97 \mathrm{k}-\mathrm{q}$ & 39 & 45.3 \\
\hline 52 & Opus & $10.1 \mathrm{e}-\mathrm{n}$ & 36 & $19.6 \mathrm{r}$ & 54 & 1.94 wx & 54 & 48.0 \\
\hline 53 & Venture & $7.05 \mathrm{o}$ & 54 & $20.35 \mathrm{rq}$ & 53 & $2.98 \mathrm{k}-\mathrm{t}$ & 38 & 48.3 \\
\hline 54 & Hystyle & 8.6 on & 53 & $21.7 \mathrm{p}-\mathrm{r}$ & 52 & $2.47 \mathrm{t}-\mathrm{w}$ & 50 & 51.7 \\
\hline 55 & $\begin{array}{l}\text { Cornell } 502 \\
\text { S.E. }\end{array}$ & $\begin{aligned} & 2.25 \mathrm{p} \\
\pm & 3.75\end{aligned}$ & 55 & $\begin{array}{r}4.5 \mathrm{~s} \\
\pm 14.71\end{array}$ & 55 & $\begin{aligned} & 1.56 \mathrm{x} \\
\pm & 0.78\end{aligned}$ & 55 & 55.0 \\
\hline
\end{tabular}

Hybrids and parents are arranged according to mean rank. Overall rank in the first column of hybrid or parent is based on mean rank. Mean rank is the mean of the three YC ranks. YC rank: yield component rank (rank of hybrid or parent for pod number, seed number, or seeds per pod); Car: 'Carson'; C 502: 'Cornell 502'; HB: 'HB 1880'; Ven: 'Venture'; Lab: 'Labrador'; Hys: 'Hystyle'. Within each yield component the means followed by different letters are significantly different $(P \leq 0.01)$ according to Duncan's multiple range test.

Table 3. Mean squares for pod number, seed number, and seeds per pod for the 10 parents and 45 hybrids of a diallel analysis when grown in a high temperature $\left(32^{\circ} \mathrm{C}\right.$ day $/ 28^{\circ} \mathrm{C}$ night $)$ or a low temperature $\left(16^{\circ} \mathrm{C}\right.$ day $/ 10^{\circ} \mathrm{C}$ night $)$ a controlled environment

\begin{tabular}{|c|c|c|c|c|c|c|c|}
\hline \multirow[b]{2}{*}{ Source } & \multirow[b]{2}{*}{ df } & \multicolumn{2}{|c|}{ Pods (no.) } & \multicolumn{2}{|c|}{ Seeds (no.) } & \multicolumn{2}{|c|}{ Seeds (no./pod) } \\
\hline & & $\begin{array}{l}\text { High } \\
\text { temp. }\end{array}$ & $\begin{array}{l}\text { Low } \\
\text { temp. }\end{array}$ & $\begin{array}{l}\text { High } \\
\text { temp. }\end{array}$ & $\begin{array}{l}\text { Low } \\
\text { temp. }\end{array}$ & $\begin{array}{l}\text { High } \\
\text { temp. }\end{array}$ & $\begin{array}{l}\text { Low } \\
\text { temp. }\end{array}$ \\
\hline Cross & 89 & $17.22^{* * *}$ & $14.85^{* * *}$ & $297.5^{\text {*** }}$ & $233.0^{* * *}$ & $1.85^{* * *}$ & $1.12^{* * *}$ \\
\hline GCA & 9 & $19.66^{* * *}$ & $42.88^{* * *}$ & $602.6^{* * *}$ & $654.6^{* * *}$ & $2.98^{* * *}$ & $4.82^{* * *}$ \\
\hline SCA & 35 & $16.77^{* * *}$ & $8.16^{*}$ & $245.4^{*}$ & $129.8^{*}$ & $1.62^{* * *}$ & $0.39^{*}$ \\
\hline $\operatorname{Rec}$ & 45 & 4.12 & 3.98 & $99.9^{*}$ & 51.89 & 0.24 & 0.14 \\
\hline Error & 108 & 4.27 & 3.70 & 83.99 & 39.90 & 0.22 & 0.15 \\
\hline
\end{tabular}

* Indicates significance at $P \leq 0.05$.

*** Indicates significance at $P \leq 0.0001$.

in other diallel parents. 'Brio', 'Carson', and 'HB 1880' were chilling tolerant and heat tolerant, and genes for these traits may be associated, linked, or pleiotropic in these parents. The unique responses of 'Labrador' and 'Cornell 502', for which tolerance or sensitivity to high and low temperatures were negatively associated, suggests these parents may possess different genes or alleles than parents with greater yield stability. Tolerance to high temperature also varied with yield components, such as for 'Barrier' and 'Venture', which both showed relatively high pod set, but relatively low seed set, in the heat treatment. This result suggests pod number and seed number under temperature stress may be under separate genetic control, an observation supported by the lack of correlation between mean pod number and mean seed number (Table 4).

The diverse responses of parental performance to temperature stress were also observed for breeding value (i.e. GCA), with significant positive and

Table 4. Pearson product-moment correlations between general combining ability (GCA) and yield component means for the 10 diallel parents when grown in a high temperature $\left(32^{\circ} \mathrm{C}\right.$ day $/ 28^{\circ} \mathrm{C}$ night $)$ or a low temperature $\left(16^{\circ} \mathrm{C}\right.$ day $/ 10^{\circ} \mathrm{C}$ night $)$ controlled environment

\begin{tabular}{llllll}
\hline & $\begin{array}{l}\text { GCA } \\
\text { seeds } \\
\text { (no.) }\end{array}$ & $\begin{array}{l}\text { GCeds } \\
\text { (no./pod) }\end{array}$ & $\begin{array}{l}\text { Mean } \\
\text { pods } \\
\text { (no.) }\end{array}$ & $\begin{array}{l}\text { Mean } \\
\text { seeds } \\
\text { (no.) }\end{array}$ & $\begin{array}{l}\text { Mean } \\
\text { seeds } \\
\text { (no./pod) }\end{array}$ \\
\hline High temperature & & & & & \\
GCA pods (no.) & 0.62 & $0.85^{*}$ & 0.61 & 0.52 & 0.32 \\
GCA seeds (no.) & & $0.68^{*}$ & $0.95^{* * *}$ & 0.29 & $0.86^{*}$ \\
GCA seeds (no./pod) & & & $0.65^{*}$ & 0.49 & 0.47 \\
Mean pods (no.) & & & & 0.42 & $0.93^{*}$ \\
Mean seeds (no.) & & & & & 0.38 \\
Low temperature & & & & & \\
GCA pods (no.) & $0.81^{*}$ & $0.55^{*}$ & 0.59 & -0.19 & 0.14 \\
GCA seeds (no.) & & $0.71^{*}$ & $0.86^{*}$ & 0.18 & 0.55 \\
GCA seeds (no./pod) & & & $0.90^{* * *}$ & $0.70^{*}$ & $0.835^{*}$ \\
Mean pods (no.) & & & & 0.547 & $0.85^{*}$ \\
Mean seeds (no.) & & & & & $0.87^{*}$ \\
\hline
\end{tabular}

*Indicates significance at $P \leq 0.05$.

*** Indicates significance at $P \leq 0.0001$. 
Table 5. General combining ability (GCA) of the 10 diallel parents for pod number, seed number, and seeds per pod when grown in a high temperature $\left(32^{\circ} \mathrm{C}\right.$ day $/ 28^{\circ} \mathrm{C}$ night $)$ or a low temperature $\left(16^{\circ} \mathrm{C}\right.$ day $/ 10^{\circ} \mathrm{C}$ night) controlled environment

\begin{tabular}{|c|c|c|c|c|c|c|}
\hline \multirow[b]{2}{*}{ Parent } & \multicolumn{2}{|c|}{ Pods (no.) } & \multicolumn{2}{|c|}{ Seeds (no.) } & \multicolumn{2}{|c|}{ Seeds (no./pod) } \\
\hline & $\begin{array}{l}\text { High } \\
\text { temp. }\end{array}$ & $\begin{array}{l}\text { Low } \\
\text { temp. }\end{array}$ & $\begin{array}{l}\text { High } \\
\text { temp. }\end{array}$ & $\begin{array}{l}\text { Low } \\
\text { temp. }\end{array}$ & $\begin{array}{l}\text { High } \\
\text { temp. }\end{array}$ & $\begin{array}{l}\text { Low } \\
\text { temp. }\end{array}$ \\
\hline Barrier & $-1.03^{*}$ & $1.42^{* * *}$ & $-3.90^{*}$ & -0.91 & $-2.09^{*}$ & $-0.48^{* * *}$ \\
\hline Brio & 0.57 & $1.38^{* * *}$ & $2.38^{*}$ & $7.37^{* * *}$ & 0.07 & $0.28^{* * *}$ \\
\hline Carson & 0.38 & -0.31 & $3.51^{*}$ & 0.35 & $0.33^{* * *}$ & $0.14^{*}$ \\
\hline Cornell 502 & $1.27^{*}$ & $-1.15^{*}$ & $5.90^{* * *}$ & $-5.50^{* * *}$ & $0.15^{*}$ & $-0.32^{* * *}$ \\
\hline CT 70 & 0.01 & -0.50 & $4.00^{*}$ & 0.67 & $0.33^{* * *}$ & $0.28^{* * *}$ \\
\hline НВ 1880 & -0.05 & 0.22 & -2.01 & 0.75 & 0.04 & 0.04 \\
\hline Hystyle & $-0.76^{*}$ & $-0.97^{*}$ & $-3.24^{*}$ & $-5.60^{* * *}$ & $-0.29^{*}$ & $-0.30^{* * *}$ \\
\hline Labrador & -0.26 & 0.04 & $-4.37^{*}$ & $3.57^{*}$ & $-0.35^{* * *}$ & $0.32^{* * *}$ \\
\hline Opus & -0.31 & $0.96^{*}$ & $-2.07^{*}$ & -1.25 & $-0.27^{*}$ & $-0.35^{* * *}$ \\
\hline Venture & 0.18 & $-1.09^{*}$ & -0.20 & 0.5336 & $0.20^{*}$ & $0.39^{* * *}$ \\
\hline Standard error & \pm 0.35 & \pm 0.27 & \pm 0.97 & \pm 0.81 & \pm 0.06 & \pm 0.04 \\
\hline
\end{tabular}

* Indicates significance at $P \leq 0.05$.

*** Indicates significance at $P \leq 0.0001$.

significant negative GCA values present in both temperature treatments (Table 5). For example, 'Cornell 502', a breeding line selected for heat tolerance, showed significant positive GCA for all of the YC under high temperature, but had a significant negative GCA for all the YC under low temperature. Parental combining ability also varied with yield components, with 'Venture' possessing significant positive GCA for seeds per pod, and significant negative GCA for pod number, under low temperature. Under low temperature, 'Barrier' had significant positive GCA for pod number, significant negative GCA for seeds per pod, and under high temperature, significant negative GCA for pod number. The variation in GCA for pod number and seeds per pod in 'Barrier' and 'Venture' provides additional evidence that these traits are under separate genetic control. The parents with the best combing ability under high temperature were 'Brio', 'Carson, 'Cornell 502', and 'CT 70', and 'Brio' under low temperature.

General combining ability could not be predicted from parental performance under temperature stress. For example, 'HB 1880' showed yield stability under both high and low temperature, but GCA was insignificant in all cases for this parent (Table 5). 'Barrier' showed high pod set under heat stress, but had significant negative GCA for this trait, and low pod set under chilling stress, with significant positive GCA for that trait. Additionally, within yield components, GCA and YC means (with the exception of seeds per pod in the low temperature treatment) were not correlated (Table 4). Parents with high yield potential under temperature stress were equally likely to have significant negative GCA as significant positive GCA for the three YC. The association of negative GCA with lines of high yield potential is a phenomenon that hinders yield improvement in common bean (Nienhuis \& Singh, 1988; Porch, 2001). These observations confirm the value of diallel analysis in choosing parents for improvement of heat tolerance in common bean. Choosing effective heat tolerant or chilling tolerant parents may require knowledge of combining ability and evaluation of segregating populations developed from the $F_{1}$ 's.

The hybrids 'Brio' $\times$ 'Cornell 502', 'Brio' $\times$ 'CT 70', 'Brio' $\times$ 'HB 1880', and 'Brio' $\times$ 'Venture' (reciprocals combined) were among the 10 highest yielding $F_{1}$ 's in both temperature treatments (Tables 1 and 2). The best overall hybrid in the high temperature treatment was 'Carson' $\times$ 'CT 70', which had the most seeds and seeds per pod (Table 1). 'Brio' $\times$ 'Cornell 502' had the highest pod number under high temperature treatment. These two hybrids, in addition to 'Cornell 502' $\times$ 'HB 1880', had significantly $(P \leq 0.05)$ higher YC means under high temperature than the highest yielding parent, 'Carson'. In the low temperature treatment no hybrids yielded significantly more than 'Brio', and six of the 10 highest yielding hybrids in the low temperature treatment had this cultivar as a parent (Table 2).

Crosses with significant positive SCA are listed in Table 6. 'Barrier' $x$ 'Venture' and 'Brio' $\times$ 'Cornell 502 ' were the only hybrids that possessed significant positive SCA for a yield component (seed number) under both temperature treatments. In general, significant positive SCA in the high temperature treatment resulted from tolerant $\times$ tolerant crosses, indicating that transgressive segregation may be observed in segregating populations generated from these crosses. Two heat tolerant $\times$ heat sensitive crosses are listed in Table 6 as having significant positive SCA under high temperature ('CT 70' $\times$ 'Labrador' and 'Carson' $\times$ 'Hystyle), though these crosses did not rank highly among all crosses for performance. These two crosses yielded better than would be expected from the GCA values of 'Labrador' and 'Hystyle', suggesting heat sensitive lines may be improved by crossing to heat tolerant lines.

There was no general trend towards association of high and low temperature stress tolerances in this analysis, both in terms of parental or hybrid performance, or GCA and SCA values. Significant GCA and SCA interaction with temperature treatment indicated that 
Table 6. $F_{1}$ hybrids from a diallel mating design with significant $(P \leq 0.05)$ specific combining ability (SCA) for pod number, seed number, or seeds per pod when grown in a high temperature $\left(32{ }^{\circ} \mathrm{C}\right.$ day $/ 28^{\circ} \mathrm{C}$ night $)$ or a low temperature $\left(16^{\circ} \mathrm{C}\right.$ day/ $10{ }^{\circ} \mathrm{C}$ night $)$ controlled environment

\begin{tabular}{|c|c|c|c|c|}
\hline \multirow[b]{2}{*}{ Pods (no.) } & \multicolumn{2}{|c|}{ High temperature $\left(32^{\circ} \mathrm{C} / 28^{\circ} \mathrm{C}\right)$} & \multicolumn{2}{|c|}{ Low temperature $\left(16^{\circ} \mathrm{C} / 10^{\circ} \mathrm{C}\right)$} \\
\hline & $\mathrm{n} / \mathrm{a}$ & $\mathrm{n} / \mathrm{a}$ & 'Barrier' $\times$ 'Opus' & $1.85^{\mathrm{a}}$ \\
\hline \multirow[t]{7}{*}{ Seeds (no.) } & 'Barrier' $\times$ 'Venture' & 9.70 & 'Barrier' $\times$ 'Venture' & 7.21 \\
\hline & 'Carson' $\times$ 'CT 70’ & 8.58 & 'Brio' $\times$ 'Cornell 502' & 6.91 \\
\hline & 'Cornell 502' × 'Hystyle' & 8.29 & ‘CT 70’ × 'Labrador’ & 0.54 \\
\hline & 'CT 70’ × 'Labrador' & 7.48 & 'Carson' × 'Opus' & 0.50 \\
\hline & 'Carson' × 'Hystyle' & 7.32 & 'Brio' $\times$ 'Venture' & 0.49 \\
\hline & 'Brio' $\times$ 'Venture' & 2.52 & 'Carson' × 'Hystyle' & 0.48 \\
\hline & & & 'Cornell 502' × 'Hystyle' & 0.48 \\
\hline \multirow[t]{3}{*}{ Seeds (no./pod) } & 'HB 1880’ × 'Venture' & 0.80 & 'Brio' × 'Cornell 502' & 0.39 \\
\hline & 'Cornell 502’ × 'HB 1880' & 0.60 & 'Barrier' × 'Cornell 502' & 0.39 \\
\hline & & & 'Barrier' $\times$ 'Venture' & 0.30 \\
\hline
\end{tabular}

${ }^{\text {a SCA value. }}$

Table 7. Mean squares for interaction between general combining ability (GCA) or specific combining ability (SCA) with temperature treatment for pod number, seed number, and seeds per pod for the 10 parents and 45 hybrids of a diallel analysis when grown in high temperature $\left(32^{\circ} \mathrm{C}\right.$ day $/ 28^{\circ} \mathrm{C}$ night $)$ or a low temperature $\left(16^{\circ} \mathrm{C}\right.$ day $/ 10^{\circ} \mathrm{C}$ night $)$ treatment in a controlled environment

\begin{tabular}{lrccl}
\hline Source & df & Pods (no.) & Seeds (no.) & Seeds (no./pod) \\
\hline GCA $\times$ treatment & 9 & $41.28^{* * *}$ & $647.97^{* * *}$ & $2.13^{* * *}$ \\
SCA $\times$ treatment & 35 & $7.12^{*}$ & 70.95 & $0.44^{* * *}$
\end{tabular}

*Indicates significance at $P \leq 0.05$.

*** Indicates significance at $P \leq 0.0001$.

combining ability under high and low temperature stress was not correlated (with the exception of seed number and SCA) (Table 7). For this reason, heat and chilling tolerance should be selected independently. Moreover, Spearman's rank-correlation coefficients between parental performance per se and GCA across temperature treatments showed no significant correlation (data not shown). However, breeding value was correlated in three cases. Under both high and low temperature treatments 'Brio' showed significant positive GCA for seed number, and 'Carson' and 'CT 70' show significant positive GCA for seeds per pod. Additionally, as mentioned previously, parental performance under heat and chilling stress was associated in lines such as 'Brio' and 'HB 1880'.

\section{Conclusions}

General and specific combining ability (GCA and SCA) were significant in both temperature treatments, indicating genetic variation for pod number, seed number, and seeds per pod under both temperature treatments was present in the diallel mating design, and that experimental conditions allowed for the detection of genetic differences among the parents and progeny. Pod number, seed number, and seeds per pod were the best indicators of yield in this study. Pod number and seed number under temperature stress may be under separate genetic control. Reciprocal effects and heterosis were not significant. Observation of insignificant heterosis suggests SCA can be utilized in selecting the best parental combinations for developing heat tolerant snap beans.

A number of interactions occurred between genotype and temperature. Parental performance under high and low temperatures was associated in some diallel parents; however, the responses of 'Labrador' and 'Cornell 502', for which tolerance or sensitivity to high and low temperatures were negatively associated, suggests different alleles, genes, and combinations of genes may be available for breeding improved cultivars. Diverse responses to temperature stress were also observed for breeding value, confirming the value of diallel analysis in choosing parents for improvement of stress tolerance in common bean. Additionally, GCA could not be predicted from parental performance. Choosing effective heat tolerant or chilling tolerant parents may require knowledge of combining ability and evaluation of segregating populations developed from the $F_{1}$ 's. Breeding value, parental performance, or both, under heat and chilling stress were associated in certain parents. 


\section{References}

Abreau, Â.F.B., M.A.P. Ramalho \& D.F. Ferreira, 1999. Selection potential for seed yield from intra- and inter-racial populations in common bean. Euphytica 180: 121-127.

Austin, R.B. \& M.S.M. Maclean, 1972. A method for screening Phaseolus genotypes for tolerance to low temperatures. J Hort Sci 47: 279-290.

Bliss, F.A., 1980. Common bean. In: W.R. Fehr \& H.H. Hadley (Eds.), Hybridization of Crop Plants, pp. 273-284. American Society of Agronomics, Crop Science Society of America, Madison, WI.

Boodley, J.W. \& R. Sheldrake Jr., 1972. Cornell peat-lite mixes for commercial plant growing. Cornell Info Bull 43: 1-8.

Chinnusamy, V., K. Schumaker \& J. Zhu, 2004. Molecular genetic perspectives on cross-talk and specificity in abiotic stress signaling in plants. J Exp Bot 55: 225-236.

Coffman, F.A., 1957. Factors influencing heat resistance in oats. Agron J 49: 368-373.

Dickson, M.H., 1967. Diallel analysis of seven economic characters in snap beans. Crop Sci 7: 121-124.

Dickson, M.H., 1971. Breeding beans, Phaseolus vulgaris L., for improved germination under unfavorable low temperature conditions. Crop Sci 11: 848-850.

Dickson, M.H., 1993. Breeding for heat tolerance in green beans and broccoli. In: C.G. Kuo (Ed.), Adaptation of Food Crops to Temperature and Water Stress, pp. 296-320. Asian Res. Dev. Center Pub. No. 93-410.

Dickson, M.H. \& M.A. Boettger, 1984a. Effect of high and low temperatures on pollen germination and seed set in green beans. J Amer Soc Hort Sci 109: 372-374.

Dickson, M.H. \& M.A. Boettger, 1984b. Emergence, growth, \& blossoming of bean (Phaseolus vulgaris) under suboptimal temperatures. J Amer Soc Hort Sci 109: 257-260.

Dickson, M.H. \& R. Petzoldt, 1987. Inheritance of low temperature tolerance in beans at several growth stages. Hort Sci 22: 481483.

El-kholy, A.S., A.E. Hall \& A.M. Mohsen, 1997. Heat and chilling tolerance during germination and heat tolerance during flowering are not associated in cowpea. Crop Sci 37: 456-463.

Evans, A., 1970. Heterosis for yield in Phaseolus vulgaris crosses. Annu Rep Bean Improv Coop 13: 52-54.

Farlow, P.J., 1981. Effect of low temperature on number and location of developed seed in two cultivars of french beans (Phaseolus vulgaris L.). Aust J Agric Res 32: 32-330.

Foolad, M.R. \& A. Bassiri, 1983. Estimates of combining ability, reciprocal effects, and heterosis for yield and yield components in a common bean diallel cross. J Agric Sci 100: 103-180.

Griffing, B., 1956. Concept of general \& specific combining ability in relation to diallel crossing systems. Aust J Biol Sci 9: 463-493.

Gross, Y. \& J. Kigel, 1994. Differential sensitivity to high temperature of stages in the reproductive development of common bean (Phaseolus vulgaris L.). Field Crops Res 36: 201-212.

Hall, A.E., 2001. Crop physiological responses to temperature and climatic zones. Crop Responses to Environment, pp. 59-82. CRC Press LLC, Boca Raton, Florida.

Hall, A.E., 2004. Comparative ecophysiology of cowpea, common bean, and peanut. In: H.T. Nguyen \& A. Blum (Eds.), Physiology and Biotechnology Integration for Plant Breeding, pp. 271-326. Marcel Dekker, Inc. New York, NY.
Inoue, Y. \& H. Suzuki, 1959. Studies on the reproductive physiology of common beans VII. The effect of temperature on the function pollen and pistil. J Hortic Assoc Jpn 28: 19-22.

Kemp, G.A., 1973. Initiation and development of flowers in beans under suboptimal temperature conditions. Can J Plant Sci 53: 623-627.

Kornegay, J., J.W. White \& O.O. de la Cruz, 1992. Growth habit and gene pool effects on inheritance of yield and common bean. Euphytica 62: 171-180.

Melo, L.C., J.B. dos Santos \& M.A.P. Ramalho, 1997. Choice of parents to obtain common bean (Phaseolus vulgaris) cultivars tolerant to low temperatures at the adult stage. Braz J Genet 20: 283-292.

Mashiringi, N.A. \& G. Harahwa, 1985. The response of twenty wheat (Triticum aestivum) varieties to growing conditions in Zimbabwe. Zimbabwe Agric J 82: 11-15.

Mumba, L.E. \& N.W. Galwey, 1999. Compatibility between wild \& cultivated common bean (Phaseolus vulgaris L.) genotypes of the mesoamerican and andean gene pools: evidence from the inheritance of quantitative characters. Euphytica 180: 105119.

Monterroso, V.A. \& H.C. Wien, 1990. Flower and pod abscission due to heat stress in beans. J Amer Soc Hort Sci 115: 631-634.

Myers, J.R. \& J.R. Baggett, 1999. Improvement of snap beans. In: S.P. Singh (Ed.), Common Bean Improvement in the Twenty-First Century, pp. 289-329. Kluwer Academic Publishers, Dordrecht, The Netherlands.

Nakano, H., T. Momonoki, T. Miyashige, H. Otsuka, T. Hanada, A. Sugimoto, H. Nakagawa, M. Matsuoka, T. Terauchi, M. Kobayashi, M. Oshiro, K. Yasuda, N. Vanichwattanarumruk, S. Chotechuen \& D. Boonmalison, 1997. 'Haibushi', a new variety of snap bean tolerant to heat stress. JIRCAS J 5: 1-12.

Nienhuis, J. \& S.P. Singh, 1986. Combining ability analysis and relationships among yield, yield components, \& architectural traits in dry bean. Crop Sci 26: 21-27.

Nienhuis, J. \& S.P. Singh, 1988. Genetics of seed yield and its components in common bean Phaseolus vulgaris L. of middle-american origin I. General combining ability. Plant Breeding 101: 143154.

Ofir, M., Y. Gross, F. Bangerth \& J. Kigel, 1993. High temperature effects on pod and seed production as related to hormone levels and abscission of reproductive structures in common bean (Phaseolus vulgaris L.). Scientia Hort 55: 201-211.

Otubo, S.T., M.A.P. Ramalho, A.F.B. Abreu \& J.B. dos Santos, 1996. Genetic control of low temperature tolerance in germination of the common bean (Phaseolus vulgaris L.). Euphytica 89: 313317.

Porch, T.G., 2001. Genetics and applications of heat tolerance in common bean. Ph.D. Dissertation, Cornell University, Ithaca, New York.

Porch, T.G. \& M. Jahn, 2001. Effects of high-temperature stress on microsporogenesis in heat-sensitive and heat-tolerant genotypes of Phaseolus vulgaris. Plant Cell Environ 24: 723-731.

SAS Institute, 1997. SAS User's Guide. SAS Institute, Cary, NC.

Shonnard, G.C. \& P. Gepts, 1994. Genetics of heat tolerance during reproductive development in common bean. Crop Sci 34: 11681175.

Singh, S.P., 1999. Integrated genetic improvement. In: S.P. Singh (Ed.), Common bean improvement in the twenty-first century, pp. 133-166. Kluwer Academic Publishers, Dordrecht, The Netherlands. 
Singh, S.P., 2001. Broadening the genetic base of common bean cultivars: A review. Crop Sci 41: 1659-1675.

Singh, S.P., H. Téran, A. Molina \& J.A. Gutiérrez, 1992. Combining ability for seed yield and its components in common bean of Andean origin. Crop Sci 32: 81-84.

Williams, J.G.K., A.R. Kubelik, K.J. Livak, J.A. Rafalski \& S.V. Tingey, 1990. DNA polymorphisms amplified by arbitrary primers are useful as genetic markers. Nucleic Acids Res 18: 6531-6535.

Yacoob, R.K. \& W.G. Filion, 1986. Temperature-stress response in maize: A comparison of several cultivars. Can J Genet Cytol 28: 1125-1131.

Zhang, Y. \& M.S. Kang, 1997. DIALLEL-SAS: A SAS program for Griffing's diallel analysis. Agronomy J 89: 176-182. 